\section{Initial presentation}

- $\mathrm{M} / 53$, intermittent retrosternal pain and dysphagia

- Gastroscopy (21/01/2014): $2 \mathrm{~cm}$ soft sub-pedunculated polypoid mass in lower oesophagus, biopsy- adenocarcinoma

- CT TAP: Distal oesophageal lesion occupying entire lumen, no lymphadenopathy, $20 \mathrm{~mm}$ right adrenal 'incidentaloma' with mild calcification and reassuring imaging characteristics

- EUS T2NO oesophageal mass

- 18FDG PET CT: high-grade metabolically active lesion in distal oesophagus (SUV-max 9.5); $2.7 \mathrm{~cm}$ nodule in right adrenal with some calcification, HU 30 (higher than lipid rich adenoma), intermediate grade increased activity (SUV-max 4.2), unlikely to be a metastasis.

- Ivor Lewis oesophagectomy and mesh repair of incisional hernia a year later without any endocrine assessment.

- Histologically: moderately differentiated adenocarcinoma, pT1b, pN0, RO, VO.

\section{Two years after;}

- CT scan for post thoracotomy pain in right side of chest: right adrenal lesion increased slightly in size, appeared necrotic- ? metastasis from his oesophageal carcinoma or a primary adrenal lesion.

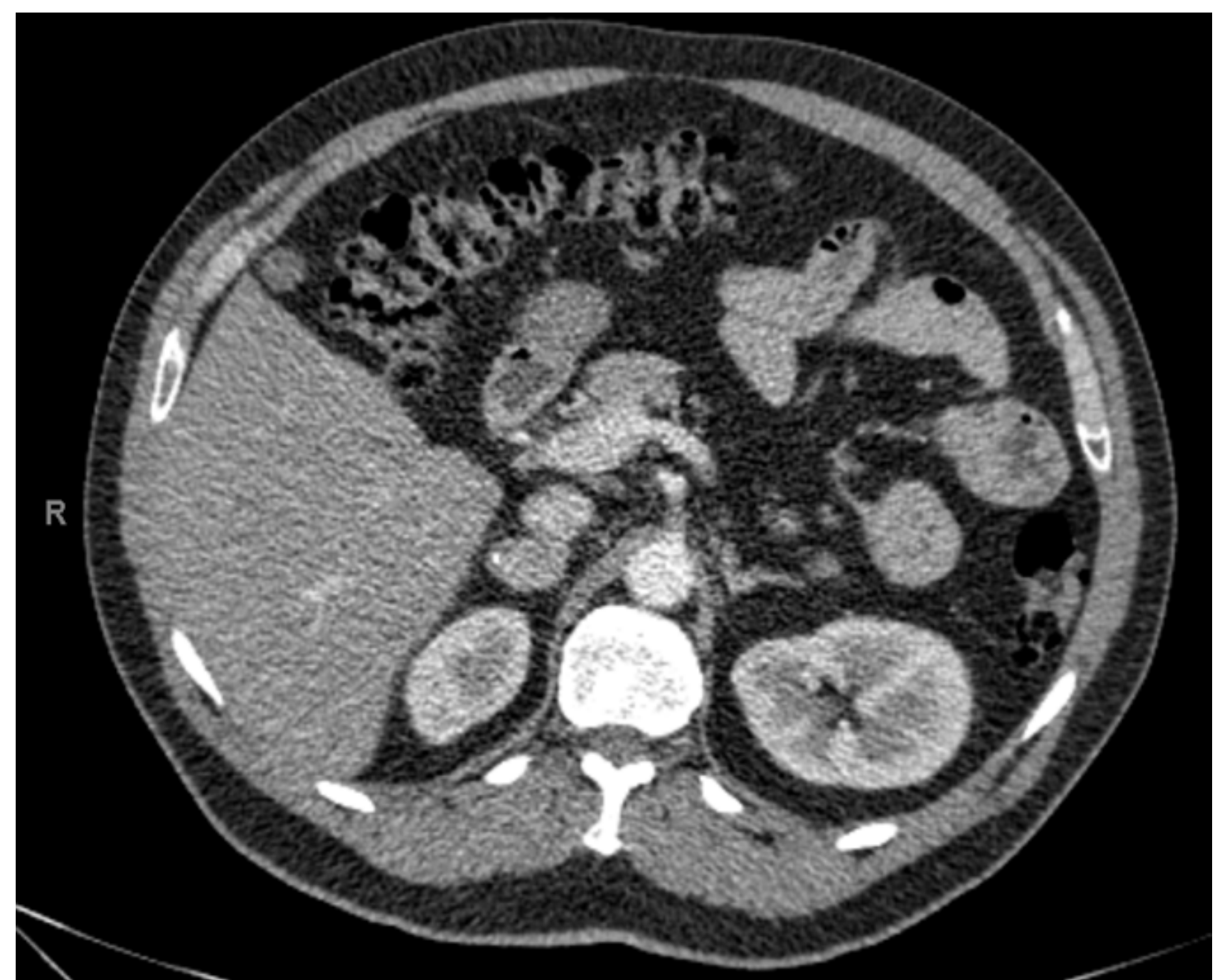

Initial

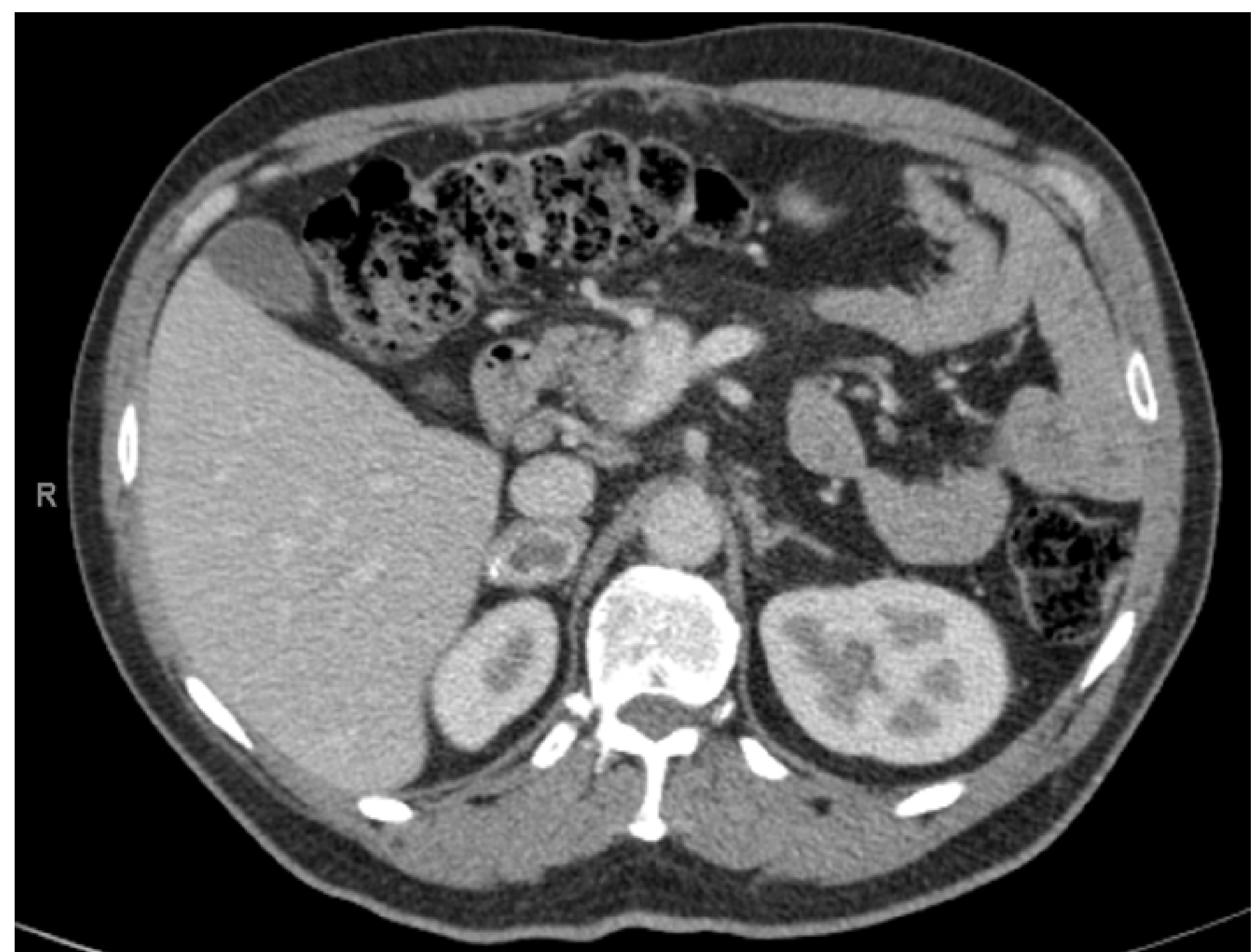

Two years later
- Referred to endocrine team- asymptomatic besides slightly reduced effort tolerance.

- 24 hour urinary free noradrenalin on three separate days were 1318, 1755 and $837 \mathrm{nmol} / 24 \mathrm{hr}$ (ref: 82-650) with normal adrenaline and dopamine.

- MIBG: Intense uptake within the gastric pull-up, normal uptake in the adrenal glands, no obvious neuroendocrine tumour elsewhere

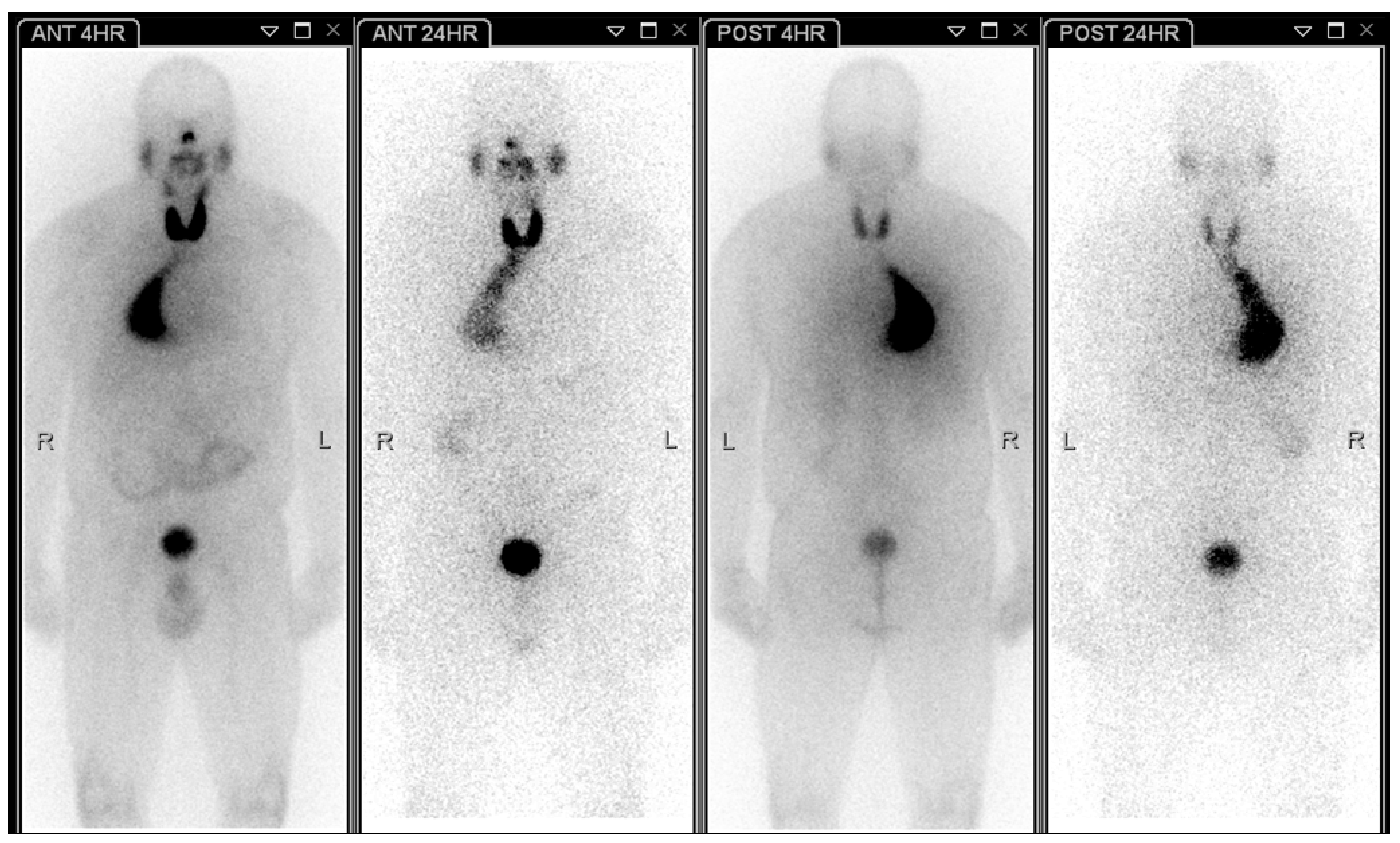

\section{References:}

1. Kantorovich V, Pacak K. Pheochromocytoma and paraganglioma. Progress in brain research. 2010;182:343-373. Mojtahedi A et al. The value of 68Ga-DOTATATE PET/CT in diagnosis and management of neuroendocrine tumors compared to current FDA approved imaging modalities: a review of literature. American Journal of Nuclear Medicine and Molecular Imaging. 2014;4(5):426-434.

Barrio M et al. The Impact of Somatostatin Receptor-Directed PET/CT on the Management of Patients with Neuroendocrine Tumor: A Systematic Review and Meta-Analysis. J Nucl Med. 2017 May;58(5):756-761. Neuroendocrine Tumor: A Systematic Review and Meta-Analysis. J Nucl Med. 2017 May;58(5):756-761.
Fonte JS et al. False-negative ${ }^{223}$-MIBG SPECT is most commonly found in SDHB-related pheochromocytoma or paraganglioma with high frequency to develop metastatic disease. Endocr Relat Cancer. 2012 Feb 13;19(1):83-93.
Biochemical investigations to confirm catecholamine hypersecretion

\begin{tabular}{|c|c|c|c|c|}
\hline & Day 1 & Day 2 & Day 3 & $\begin{array}{l}\text { Reference } \\
\text { range }\end{array}$ \\
\hline \multicolumn{5}{|l|}{ Plasma metanephrines } \\
\hline Normetanephrine (pmol/L) & 2657 & 3298 & 3037 & $120-1180$ \\
\hline Metanephrine (pmol/L) & 301 & 333 & 293 & 80- 510 \\
\hline 3-methoxytyramine (pmol/L) & $<180$ & $<180$ & $<180$ & $<180$ \\
\hline \multicolumn{5}{|l|}{$24 \mathrm{hr}$ urinary catecholamines } \\
\hline Free noradrenaline (nmol/col) & 1478 & 2016 & - & $82-650$ \\
\hline Total normetadrenaline ( $\mu \mathrm{mol} / \mathrm{col})$ & 8.6 & 9.2 & - & $0-4.9$ \\
\hline \multicolumn{5}{|l|}{ Plasma normetanephrine (pmol/L) } \\
\hline Sitting & 3298 & & & \\
\hline After $\mathbf{3 0}$ minute supine rest & 3037 & & & \\
\hline
\end{tabular}

- 68Ga-DOTATATE scan: Intense DOTATATE uptake in right adrenal nodule $(2.8 \times 2.1 \mathrm{~cm})$, higher than expected physiological activity (SUVmax 30, cf left adrenal with normal configuration had SUVmax 16), no DOTATATE avid disease elsewhere, $12 \mathrm{~mm}$ nodule in right lung oblique fissure
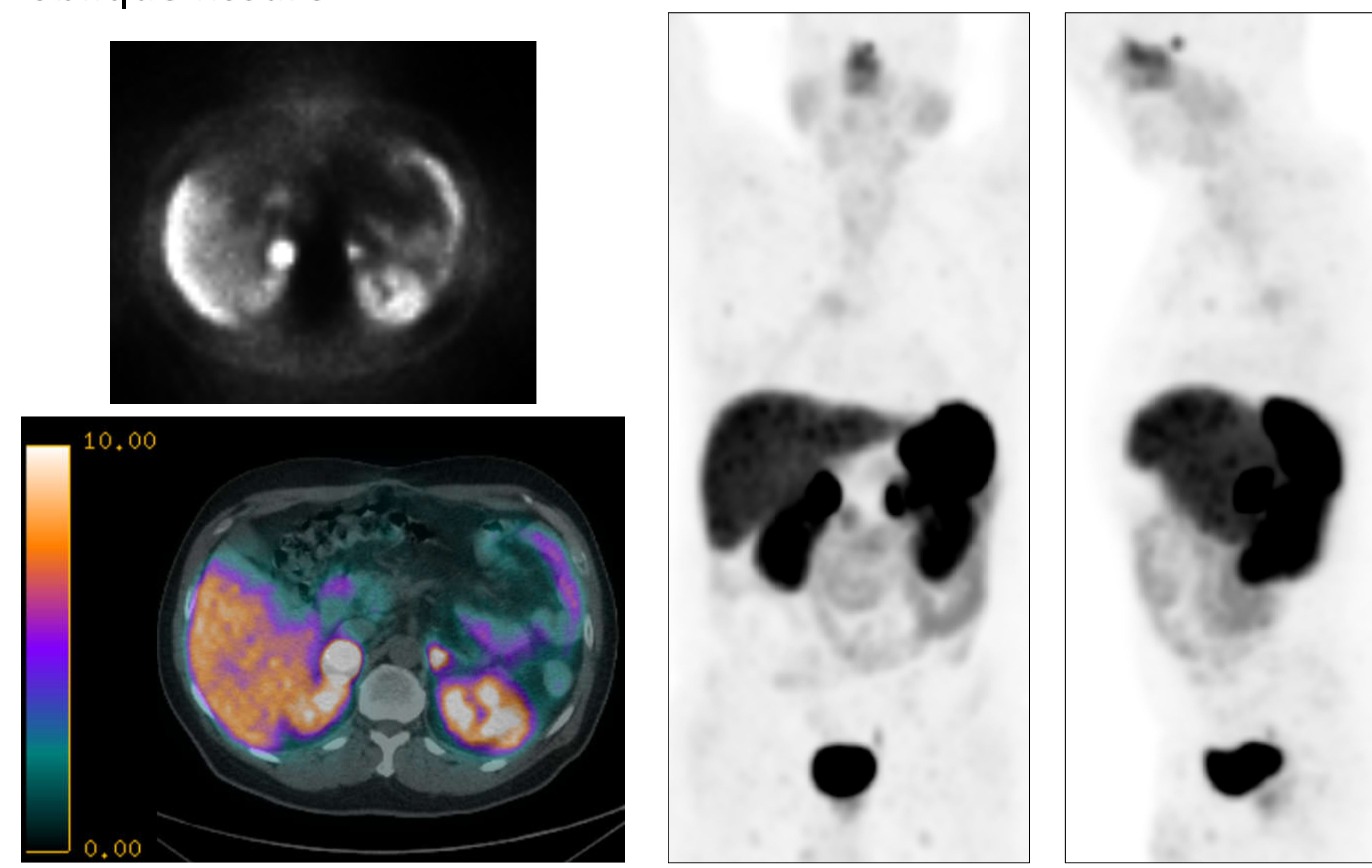

- Right adrenalectomy was performed after appropriate alpha and beta blockade.

- Histology confirmed phaeochromocytoma (immunohistochemistry positive with chromogranin and synaptophysin) with PASS (Phaeochromocytoma of the Adrenal gland Scoring Scale) score of two.

\section{Discussion:}

This case reminds us of certain interesting points:

- Although most adrenal phaeochromocytomas secrete both norepinephrine and epinephrine, about a third exclusively produce norepinephrine and a much smaller proportion exclusively produce epinephrine [1].

- DOTATATE scan is generally found to be more sensitive and specific than MIBG scan for diagnosis of NETs [2]. In a recent meta-analysis of 14 studies, change in management occurred in 44\% (range: $16-71 \%$ ) of NET patients after SSTR PET/CT. In 4/14 studies SSTR PET/CT was performed after an ${ }^{111}$ In-Octreotide scan. In this subgroup additional information by SSTR PET/CT led to a change in management in $39 \%$ (range: $16-71 \%$ ) of patients [3].

- There is some association in literature linking false negative MIBG scans with SDHB mutations, high frequency to develop metastatic disease, extra-adrenal location and hypersecretion of normetanephrine or norepinephrine [4].

- Oesophageal uptake in MIBG scan proved to be a red herring. Red herrings in isotope scans are so common that they are no longer red!

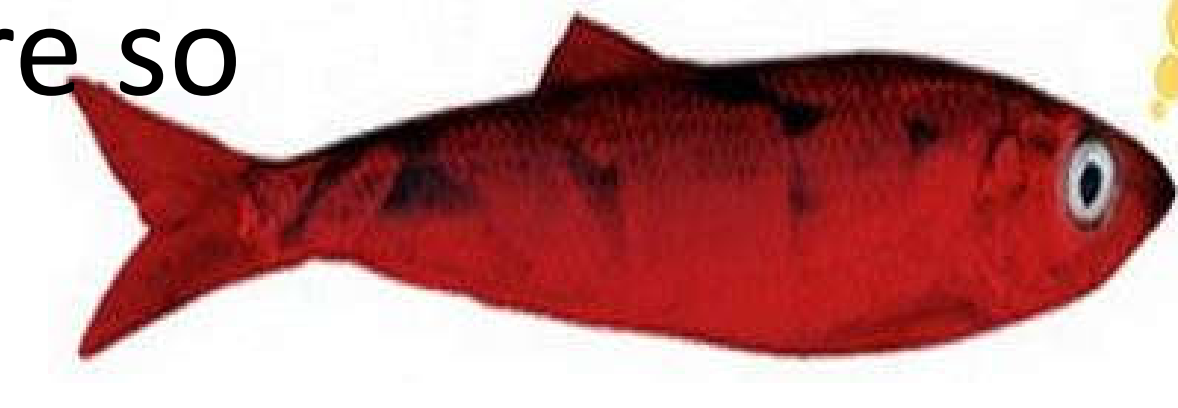

